This article was downloaded by: [B-on Consortium - 2007]

On: 15 September 2009

Access details: Access Details: [subscription number 908038078]

Publisher Routledge

Informa Ltd Registered in England and Wales Registered Number: 1072954 Registered office: Mortimer House, 37-41 Mortimer Street, London W1T 3JH, UK

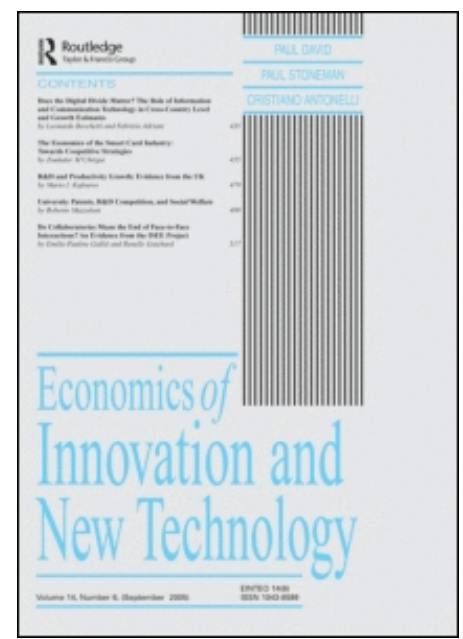

Economics of Innovation and New Technology

Publication details, including instructions for authors and subscription information:

http://www.informaworld.com/smpp/title content=t713641545

\title{
Simulating the prospects of technological catching up
}

José Castro Caldas a; Manuel Mira Godinho b; Ricardo Paes Mamede ${ }^{c}$

${ }^{a}$ Centro de Estudos Sociais, Colégio de S. Jerónimo, Coimbra, Portugal ${ }^{\mathrm{b}}$ UECE and ISEG, Rua Miguel Lupi, Lisboa, Portugal ${ }^{\mathrm{c}}$ Dinâmia and ISCTE - Lisbon University Institute, Lisboa, Portugal

Online Publication Date: 01 January 2009

To cite this Article Caldas, José Castro, Godinho, Manuel Mira and Mamede, Ricardo Paes(2009)'Simulating the prospects of technological catching up',Economics of Innovation and New Technology, 18:6,565 - 586

To link to this Article: DOI: $10.1080 / 10438590802233651$

URL: http://dx.doi.org/10.1080/10438590802233651

\section{PLEASE SCROLL DOWN FOR ARTICLE}

Full terms and conditions of use: http://www.informaworld.com/terms-and-conditions-of-access.pdf

This article may be used for research, teaching and private study purposes. Any substantial or systematic reproduction, re-distribution, re-selling, loan or sub-licensing, systematic supply or distribution in any form to anyone is expressly forbidden.

The publisher does not give any warranty express or implied or make any representation that the contents will be complete or accurate or up to date. The accuracy of any instructions, formulae and drug doses should be independently verified with primary sources. The publisher shall not be liable for any loss, actions, claims, proceedings, demand or costs or damages whatsoever or howsoever caused arising directly or indirectly in connection with or arising out of the use of this material. 


\title{
Simulating the prospects of technological catching up
}

\author{
José Castro Caldas ${ }^{\mathrm{a}}$, Manuel Mira Godinho ${ }^{\mathrm{b}}$ and Ricardo Paes Mamede ${ }^{\mathrm{c} *}$ \\ ${ }^{a}$ Centro de Estudos Sociais, Colégio de S. Jerónimo, Apartado 3087, 3301-401 Coimbra, Portugal; \\ ${ }^{b}$ UECE and ISEG, Rua Miguel Lupi, 1200 Lisboa, Portugal; ${ }^{c}$ Dinâmia and ISCTE - Lisbon \\ University Institute, Avenida das Forças Armadas, 1649-026 Lisboa, Portugal
}

(Received 11 January 2007; final version received 28 October 2008)

\begin{abstract}
Local increasing returns associated with static and dynamic scale effects, knowledge spillovers, polarization effects and the distance that separates different regions are among the most important driving forces behind the dynamics of economic and technological convergence. This paper puts forward a computational simulation model that seeks to integrate these factors. The modelling exercise was designed in order to achieve a better understanding of the relationship between the aspects underlying the specific trajectories of regional technological accumulation and the aggregate convergence/divergence patterns stemming from these trajectories. In particular, the role of history and geography in the dynamics of technological convergence is emphasized.
\end{abstract}

Keywords: technological convergence; knowledge spillovers; polarization effects

JEL Classification: $\mathrm{O} 33$

\section{Introduction}

The analysis of economic convergence is an issue that has attracted much attention from economists. Both theoretical and empirical approaches have sought to provide a rationale and an explanation for the dynamics of inter-regional convergence processes. Empirical analyses have shown that, for different historical periods and for different groups of regions or countries, quite different patterns of convergence are observable. These analyses indicate that, in certain cases, the gap between more and less advanced regions has tended to diminish, while, in other cases, steady inter-regional divergence has shown itself to be a persistent feature. Probably, partly as a result of such contrasting empirical patterns, the theoretical literature on convergence - which has developed significantly in recent decades - has shown itself to be divided in accepting or rejecting the possibility of inter-regional convergence.

Our paper develops a computational simulation model that attempts to provide a synthesis of some of the major arguments stemming from both the theoretical and empirical literatures. The aim of the modelling exercise is to achieve a better understanding

*Corresponding author. Email: ricardo.mamede@iscte.pt

ISSN 1043-8599 print/ISSN 1476-8364 online

(C) 2009 Taylor \& Francis

DOI: $10.1080 / 10438590802233651$

http://www.informaworld.com 
of the relationship between the aspects underlying the specific trajectories of regional technological accumulation and the aggregate convergence/divergence patterns stemming from such trajectories.

Section 2 of this paper contains a discussion of some of the main theoretical contributions and a brief description of the results presented by some recent empirical studies on convergence. In Section 3, the computational simulation model is presented. Then, in Section 4, we concentrate on a thorough examination of the results of the computational simulations, for different values of the critical parameters in the model. The paper ends with a brief final section, in which major conclusions are drawn and prospects for further work outlined.

\section{Background: theories and empirical facts}

Theoretical considerations about inter-regional economic convergence can be broadly divided into three main groups. The first group, which might be referred to as 'unconditional convergence theories', adheres to a generic idea of observing regions as open systems. Such openness allows for the dissemination of certain critical assets that may gradually bring about equalization in economic performances. In the case of conventional neo-classical trade and growth theories, and assuming the usual caveats, namely that there are no barriers to factor mobility, convergence is understood to arise from the flows of labour and capital to regions with higher wages or profit rates. ${ }^{1}$ In the case of traditional 'technology gap' approaches (as put forward by Posner (1961) or Gerschenkron (1962)), the assumption is that backward regions (or countries) are able to absorb the technology previously developed by the leading countries. This diffusion provides backward regions with the capacity of increasing their rate of economic growth and converging towards the world frontier without incurring, in terms of $R \& D$, costs similar to those previously paid by the advanced regions.

Opposite views, which might generally be considered as 'divergence theories' of growth, have been put forward by authors such as Kaldor. As a firm opponent of the neo-classical tradition, Kaldor (1957) rejected the idea that integration between different economies eliminates income differentials. In short, his model emphasized the interdependence between the factors of growth (investment, innovation and population growth) and the processes of cumulative causation. The latter are mainly related to what he termed the Verdoorn law - the idea that productivity growth is dependent on output growth, due to the presence of economies of scale (both static and dynamic). Kaldor also noted that, in the course of economic integration, emigration to the richer regions tends to depress local demand in the poorer regions, while markets in the former regions enlarge rapidly as a result of agglomeration economies. ${ }^{2}$ Thus, Kaldor explicitly considered the importance of increasing returns in the explanation of growth processes acknowledging the work of Allyn Young, whose impact on later theories of endogenous growth can hardly go unnoticed (Romer 1986).

The last strand of literature, which goes by the name of 'conditional convergence theories', assumes that certain pre-requisites - such as the existence of a social capability or the need for technological congruence between the backward and the advanced nations (Abramovitz 1986) - are necessary for successful catching up. By acknowledging the validity and merits of some of the arguments arising from the two groups of approaches outlined above, this strand of literature has tended to include most of the recent contributions on convergence analysis. ${ }^{3}$ In this vein, the technology gap literature (Fagerberg 1987, 1994; Verspagen 1991) assumes that both innovation and diffusion processes must be accounted for in the analysis of economic convergence. But, while the latter process tends 
to reduce gaps, the former tends to widen them. In the end, the question that arises is which of these processes will eventually predominate. ${ }^{4}$ The extent of the ability of the catching-up countries or regions to absorb innovative technology is therefore a critical factor.

Relatively similar views have been put forward by authors writing from neoSchumpeterian and evolutionary perspectives. For example, in an analysis of the post-World War II convergence of some European countries and Japan towards the 'best-practice' levels of the USA, Pavitt (1985) stressed that catching up is a long-term endeavour, resulting primarily from a path-dependent process of sequential accumulation of technological knowledge. Evolutionary theory also allows us to understand the critical role performed by certain institutions (governance, education, science, finance) and the influence of the structure of interactions on growth differentials.

The idea of conditional convergence is also clearly to be found in the "new growth theories', which assume that a significant negative correlation between growth rates in a given period and initial income levels depends, among other aspects, on the existence of adequate literacy rates or appropriate $R \& D$ outlays in the less advanced regions.

Having very briefly sketched out the main strands of the theoretical literature written on the convergence topic, one question arises: how do these theories match up to empirical findings? As was said in the introductory section, different convergence patterns are to be found in relation to both the historical periods considered and the sets of regions selected for analysis. Nevertheless, despite this variance in the patterns found by empirical analysis, it is also clear from the results reported by existing studies, namely those referring to the European Union regions and countries (Abraham and van Rompuy 1995; Armstrong 1995; Barro and Sala-i-Martin 1992; Fagerberg, Verspagen, and Caniëls 1997; Cappelen, Fagerberg and Verspagen 1999; Neven and Gouyette 1995), that certain 'hard facts' may be identified as representing general characteristics of these processes.

The indicators normally used by empirical analysis to account for the convergence trends include GDP per capita and labour productivity. The story that both indicators tell us about convergence in the EU is approximately the same. Excluding the cohesion countries, for which regional series have only existed since 1980, these indicators show that economic inter-regional convergence progressed significantly until 1980. Since then, and continuing with the most recent figures published in the second half of the 1990s, convergence has stalled and dispersion has remained approximately unchanged. The inclusion for the period after 1980 of the regions from the cohesion countries, namely those from Ireland, Portugal and Spain, shows a relatively different story. For this larger sample of regions, inter-regional economic dispersion has continued to decline since 1980. However, one important qualification must be made with regard to the trend observed in the larger sample: the rate of convergence in the period after 1980 is much more significant when comparing EU countries than when comparing the total number of regions included in those countries.

The conclusions that one may draw from these facts are two-fold. First, opportunities for economic convergence seem to be declining in the more recent period in comparison with the decades before 1980. Secondly, while European integration and structural funds seem to have had a positive overall effect on the cohesion countries, inter-regional disparities within those countries have been increasing. In relation to the theoretical debate between the three contrasting strands of literature referred to above, these facts seem to lend support to the 'conditional convergence' or even to the 'divergence' views. It is also evident that while some factors are helping certain regions (Ireland, Lisboa e Vale do Tejo, Catalonia, Athinai, etc.) to develop and move closer to the more advanced regions, there are other factors that are retarding, or at least inhibiting, the development of a large number of other regions, both within and outside the group of cohesion countries. Furthermore, as far as 
empirical analyses are concerned, one aspect that needs to be clarified is what causes the trends observed in the EU.

At least two factors must be taken into account when trying to develop a rationale for the trends observed since the 1980s: the emergence and dissemination of a significant set of ICT-based technologies and activities and the increasing openness and integration of economies, with the intensification of both the Europeanization and globalization processes. These two factors interact together and have proved to be mutually reinforcing. The regions that have been more successful in developing, disseminating and using the newer technologies are those that display and adequately exploit certain critical masses of resources and competences in specific scientific, technological and organizational domains. ${ }^{5}$ Empirical observation also shows that, in order to be effective, these critical masses need to reach certain thresholds in terms of size. ${ }^{6}$ On the other hand, the decline in the relative importance of national borders has led to the concentration of key management and technical activities in a smaller number of centres of administration, co-ordinating activities across wider areas than before. The emergence of 'core-nodes' in economic and technical networks may be seen as polarization phenomena helping to retard the long-term advances towards convergence.

At this juncture, it is useful to bring into the discussion certain empirical facts about the economic disparities between the EU regions. The available data show that over the last few decades the regions with the highest levels of GDP per capita are consistently those regions that have both the highest populations and the highest wealth densities (measured in inhabitants $/ \mathrm{km}^{2}$ and GDP $/ \mathrm{km}^{2}$, respectively). The very high correlation measures to be seen in Table 1 show a steady pattern in the concentration of people, economic activities and incomes resulting from long-term trajectories that have continued up to the present day. Richer regions tend to attract more people because they are richer, and they are richer because they have attracted more people.

These empirical data indicate that some regions have taken advantage of some sort of polarization effects. It becomes clear that in the presence of such effects, similar rates of local efforts (investment in tangible and intangible capital, etc.) do not necessarily lead to the same outcomes in terms of growth. On the contrary, the regions where these effects are more intense seem to be able to benefit from various sources of increasing returns that lead to systematic advantage and eventually to divergence. The understanding of the possible sources of these increasing returns is therefore critical for understanding why in some circumstances divergence may be a more plausible result than convergence.

Static and dynamic economies of scale are among such sources, and they relate to conditions that are both internal and external to the firm, with dynamic economies of scale going well beyond internal learning by doing. In fact, learning by interacting or by screening and observing are also important sources of efficiency improvement. Economies of scope are another crucial element in this process, relying simultaneously on static economies of

Table 1. Correlation between GDP per capita in pps, population density and $\mathrm{GDP} / \mathrm{km}^{2}$ in 76 European regions (1996).

\begin{tabular}{lccc}
\hline & GDP per capita in pps & Population density & GDP $/ \mathrm{km}^{2}$ \\
\hline GDP per capita in pps & 1.000 & & \\
Population density & $0.521^{*}$ & 1.000 & \\
GDP $/ \mathrm{km}^{2}$ & $0.454^{*}$ & $0.973^{*}$ & 1.000 \\
\hline
\end{tabular}

Note: The source of the information for these calculations is the Eurostat New Cronos database. *Correlation is significant at the 0.01 level (two-tailed). 
scale and on the increase in the potential customer base that firms may obtain by providing a wider choice of products. Finally, there is a range of increasing returns deriving only from the contextual conditions of the firm. This is the case with agglomeration economies, but also network and knowledge spillovers (Arrow 1962; Griliches 1994; Jaffe 1996; Nelson 1959).

All these sources of increasing returns are dependent, in one way or another, on the conditions under which firms operate: aspects such as market size, sophistication of buyers, density and intensity of purchasing power, innovative competitors in the neighbourhood, advanced infrastructures, etc. Most of these aspects are, in fact, much more intense in the more advanced regions. Furthermore, as far as knowledge spillovers are concerned, their effectiveness tends to be much higher over shorter geographical distances. The work of Jaffe, Trajtenberg and Henderson (1993) illustrates this point. ${ }^{7}$ Also for Europe, similar results have been obtained by Maurseth and Verspagen (1998). ${ }^{8}$ The results of these studies, which consider knowledge spillovers in terms of patent citations, are similar to those observed in case studies of certain innovative areas and clusters, where the potential of locally generated spillovers seems to decline swiftly as geographical distance increases.

In short, from this brief discussion of both the major conceptual approaches and empirical analyses and facts, we can conclude that the convergence process stems from the interplay of a significant number of factors. It is now evident that even when similar efforts are made, different regions might reach different outcomes. This happens because certain polarization effects arise, allowing for the development of some sort of increasing returns trajectories in the more advanced regions. The divergence that derives from this is partially compensated by the diffusion of knowledge that tends to be of greater benefit to the less developed regions. The pattern of interaction that arises between different regions is therefore important. Such a pattern depends mainly on geographical distance, but also on the cultural, economic and technological distances between regions. Finally, there are other aspects, relating to uncertainty and chance, which might also affect the growth trajectories and the catching-up prospects of the less-favoured regions.

\section{General outline of the model}

The aim of the modelling exercise is to achieve a better understanding of the relationship between the factors underlying the specific trajectories of regional technological accumulation and the aggregate convergence/divergence patterns stemming from those trajectories. The exercise that will be carried out concentrates on the dynamics and spatial distribution of a variable we called 'technological capability', the assumption being that this variable is a driving force of processes whose results can be measured in terms of productivity.

The model building started with the identification of the main processes associated with the factors that determine the acquisition and diffusion of 'technological capability'. The model assumes that two dimensions are involved in the development of technological capability in time and space: (a) local production of knowledge and (b) diffusion of knowledge among different 'local units' (or 'regions').

In order to model the local component (a), we abstract from the interaction between 'regions'. Knowledge creation and acquisition at the local level depend on a multitude of factors, ranging from the 'endowment and accumulation of factors' to 'social capability', and 'purposive R\&D efforts'. Essential to the model is the assumption that scale and polarization effects result from the combination of these factors, so that the local accumulation of the technological capability may be modelled as an exponential process.

In order to model component (b), we concentrate on the interaction between local units. Diffusion is mostly a consequence of learning and depends on both the opportunities for 
interaction enjoyed by the different local units and the technological gap between them. Interaction takes many different forms (from trade to direct investment or patent licensing) and occurs in various spaces simultaneously (the economic, geographical and cultural spaces, as well as the knowledge space, among many possible such spaces). In the model, a single topology is assumed for all spaces, and distance in this abstract unidimensional space is understood as determining the different local units' opportunities for interaction. When these units interact, knowledge diffusion may occur if the size of the technological gap between the interacting units allows for 'communication'. Another essential element in the model is that the probability of interaction decreases with distance in space (whatever space means).

The local units may be taken as regions defined by a location on a grid (a map) and by a given level of technological capability. This grid is not a regular one, since it is limited at its borders and may contain empty nodes. In the model, the regions whose technological capability was somehow pre-defined meet randomly pair-wise from time to time (with these interactions being less probable for partners that are distant in space). It is through these interactions that regions may learn from each other if the 'technological gap' between them is not too large, thus increasing their technological capability. Regardless of random encounters, technological capability also grows as a result of the local factors acting upon it, in accordance with process (a) outlined above.

\subsection{The computational implementation of the model}

Models of technological convergence encompassing the main ingredients discussed above - namely, the relation between local development of technological capacity and the diffusion of knowledge between interdependent economies - have been developed using the more standard analytical modelling tools, with the aim of explaining specific regularities found in the data (for a survey, see Klenow and Rodríguez-Clare (2005)). The model here used is instead a computer simulation program, the basic structure of which consists of a cellular automaton. Cellular automata are particularly adequate for our purposes, allowing us to represent (both graphically and numerically) regions interacting with each other, thus simplifying the analysis of some properties emerging from the whole system of interactions that could hardly be anticipated from the basic rules guiding the evolution of each region, without the need to identify beforehand the conditions for a steady state (as is the case with the more traditional analytical models).

Thus, in its basic structure, our model considers $n$ cells, representing regions or empty spots, located on a dim $\times \operatorname{dim}^{\text {matrix }}{ }^{9}$ (a map). Associated with each cell is a real number representing the level of technological capability (TC).

Each cell is represented on the computer screen by colours corresponding to ranges of relative technological capability, as follows:

white $\left(\mathrm{TC}_{i j}=0\right.$, corresponding to an empty spot);

$$
\begin{aligned}
& \text { grey }\left(0<\mathrm{TC}_{i j} \leq \frac{2}{5} \overline{\mathrm{TC}}\right) ; \\
& \text { green }\left(\frac{2}{5} \overline{\mathrm{TC}}<\mathrm{TC}_{i j} \leq \frac{4}{5} \overline{\mathrm{TC}}\right) ; \\
& \text { blue }\left(\frac{4}{5} \overline{\mathrm{TC}}<\mathrm{TC}_{i j} \leq \frac{6}{5} \overline{\mathrm{TC}}\right) ;
\end{aligned}
$$




$$
\begin{aligned}
& \operatorname{red}\left(\frac{6}{5} \overline{\mathrm{TC}}<\mathrm{TC}_{i j} \leq \frac{8}{5} \overline{\mathrm{TC}}\right) ; \\
& \text { yellow }\left(\mathrm{TC}_{i j}>\frac{8}{5} \overline{\mathrm{TC}}\right) .
\end{aligned}
$$

In all cases $\overline{\mathrm{TC}}=\sum_{i=1}^{\operatorname{dim}} \sum_{j=1}^{\operatorname{dim}} \mathrm{TC}_{i j} / M$, where $M$ is the total number of non-zero elements of the matrix.

The simulation is initiated by assigning integer technological capability values in the range [1,9] to each cell, either randomly sampled out from a uniformly distributed population or user-defined. Any two-dimensional map (i.e. one that is limited at the borders) may be created, with the possibility of using white cells (nil TCs) to represent empty spots.

In each time step of the simulation, a pair of non-white regions in the map is randomly chosen for interaction. Partners in interaction might be, in principle, any pair of regions in the map. However, the process by which these partners are selected is such that the probability of choosing regions that are distant from each other on the map is lower (depending on a parameter) than the probability of choosing ones that are closer together.

In order to ensure that close neighbours interact with higher probability, the interacting regions were selected as follows. Given the randomly chosen region ${ }_{i j}$, and in order to determine the co-ordinates of the interacting partner of region ${ }_{i j}$, two randomly distributed variables dist $_{i}$ and dist $_{j}$ are sequentially sampled using the function dist $=$ $\left\lfloor\operatorname{dim} \times\left(1-r^{\mathrm{SD}}\right)\right\rfloor$, where $r$ is a uniformly distributed random variable between zero and one and the 'spatial distance' parameter SD $\in] 0,1]$. The shape of the distribution of dist for different parameters SD when $\operatorname{dim}=40$ is illustrated in Figure 1. Next, two new uniformly distributed random variables between zero and one, $p$ and $p^{\prime}$ are sampled; if $p \leq 0.5$, then the interacting partner's co-ordinate $k$ is $i+$ dist $_{i}$, or else, if $p>0.5, k=i-$ dist $_{i}$; if $p^{\prime} \leq 0.5$, the interacting partner's co-ordinate $l$, is $j+$ dist $_{j}$, or else, if $p^{\prime}>0.5, l=j-$ dist $_{j}$, thus obtaining the co-ordinates of region ${ }_{k l}$, the interacting partner of region $_{i j} .{ }^{10}$

\section{Distribution of dist for SD $=0.03$}

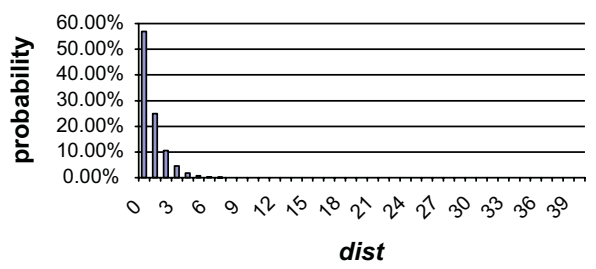

(a)

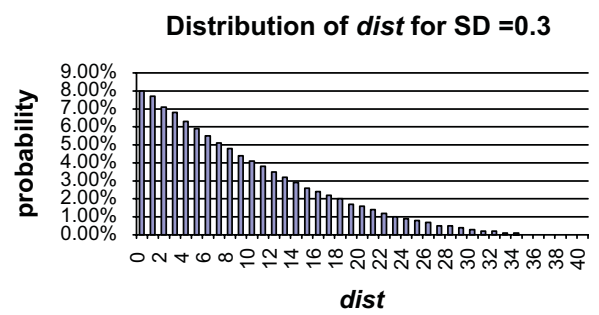

(c)

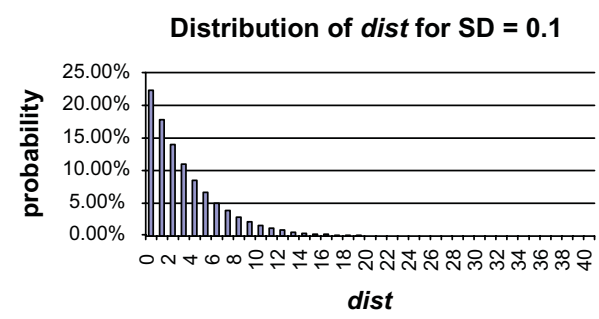

(b)

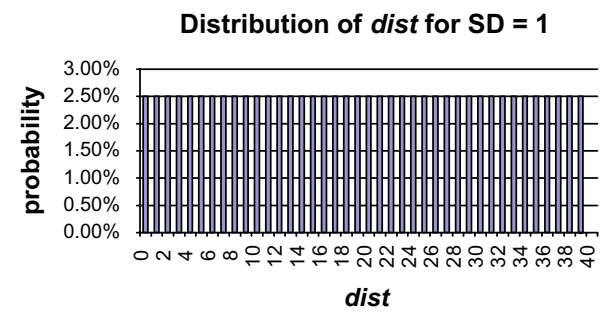

(d)

Figure 1. Shape of the distribution of dist for different parameters SD, when dim $=40$. 
With a pair of regions, their interaction is seen as possibly giving rise to diffusion ('spillovers') in both directions. Here the point was therefore reached at which the model concentrates on the process (b) described above. Then, if

$$
\frac{\max \left\{\mathrm{TC}_{i j}, \mathrm{TC}_{k l}\right\}}{\min \left\{\mathrm{TC}_{i j}, \mathrm{TC}_{k l}\right\}}<\mathrm{TT},
$$

where TT (technological threshold) is the threshold above which no communication is possible, spillovers in both directions are computed,

$$
\begin{aligned}
& \text { spillover }\left(\text { region }_{k l} \rightarrow \text { region }_{i j}\right)=\mathrm{TC}_{k l} \times \mathrm{DF} \\
& \text { spillover }\left(\text { region }_{i j} \rightarrow \text { region }_{k l}\right)=\mathrm{TC}_{i j} \times \mathrm{DF} \text {, }
\end{aligned}
$$

where DF is the diffusion factor, which can be fixed at different values. ${ }^{11}$

Subsequently the technological capabilities are updated,

$$
\begin{aligned}
\mathrm{TC}_{i j(t+1)} & =\mathrm{TC}_{i j(t)}+\operatorname{spillover}\left(\operatorname{region}_{k l} \rightarrow \operatorname{region}_{i j}\right)_{(t)} \\
\mathrm{TC}_{k l(t+1)} & =\mathrm{TC}_{k l(t)}+\operatorname{spillover}\left(\operatorname{region}_{i j} \rightarrow \operatorname{region}_{k l}\right)_{(t)} .
\end{aligned}
$$

After every 300 time steps of the simulation, ${ }^{12}$ the technological capability of each region is updated in terms of the local factors acting upon it, in accordance with process (a) outlined above. For every region the growth induced by local accumulation of knowledge is given by, $\mathrm{TC}_{(t+1)}=\mathrm{TC}_{(t)}^{1+\mathrm{IR}}$, with $\mathrm{IR} \geq 0$ as the increasing returns factor, equal for all regions and constant through the simulation.

- The initialization includes the setting up of the following parameters of the simulation: spatial distance factor (SD); technological threshold (TT); diffusion factor (DF); increasing returns factor (IR); limit number of time steps $(N)$.

The procedure may be summarized as follows:

Procedure catch;

Begin

set parameters

set $T C$ values

For $\mathrm{i}=1$ To N Do

Begin

select a pair of regions

compute spillovers

End

If $(\mathrm{N} \bmod 300)=0$ Then increase $T C$ values

\section{End.}

The initial and final values of the technological capability are recorded for statistical analysis. During the simulation, coloured dots representing the relative technological capability of the regions in every time step are drawn on the computer screen, so that the configuration patterns of the map may be observed as they emerge. 


\section{Analysing the simulation's results}

As we have just seen in Section 3, when initializing the simulation one has to define values for five parameters: ${ }^{13}$ spatial distance factor, technological threshold, diffusion factor, increasing returns factor and the stopping criteria ( $N$ is the total number of time steps of the simulation).

In order to analyse the main features of the model, we reduced the parameter space by fixing the number of time steps $N$ at 100,000 and the value of the diffusion factor DF at 0.05 . The choice of the number of time steps was based on the fact that after $N=100,000$ most of the experiments show a strong stability in their basic features. As far as the diffusion factor was concerned, we ran a number of experiments and concluded that the model was robust for a wide range of values of this parameter. The choice of 0.05 as a fixed value was, however, arbitrary. Analysis of the influence of this DF parameter on the final results therefore deserves further investigation.

Taking the values of both the number of time steps and the diffusion factor parameters as given, we ran close to 100 experiments for different values of the other three parameters. This procedure was carried out in several successive steps. After analysing the effects of several values for each of the three 'operational' variables on a ceteris paribus basis, we crossed the following values of the three parameters:

- spatial distance factor (SD): 0, 0.02, 0.2, 0.8 and 1;

- technological threshold (TT): $1,1.25,2.5$, and 10 million; ${ }^{14}$

- increasing returns factor (IR): 0, 0.0001, 0.0005, 0.001, 0.003 .

Furthermore, we tested for the effects on these results of using different random generator seeds (different values of $r$ ), as well as different spatial configurations.

The outputs of the experiments were both statistical and graphical - the coefficient of variation of the regions' technological capabilities (which is taken as an indicator of convergence/divergence between regions), the histogram of the distribution of technological capabilities and the final distribution of the coloured dots in the map.

In the following sections, we present the main results stemming from the analysis of those outputs, in terms of convergence/divergence patterns. We start by discussing the impact of changing the values of the three parameters (SD, TT and IR), which will help us to understand their role in shaping the results of the experiments. Then we analyse the factors giving rise to processes of catching up, forging ahead and falling behind. Finally, in the last two sections of this part, we pay special attention to the role of history and space configuration in shaping those processes.

\subsection{The impact of changing the values of the parameters}

In this section, we restrict the analysis of the simulation's results to three operational parameters - SD, TT and IR. As we will see below, even with such a low number of parameters, the model can give rise to some interesting, if not surprising, results. Before this, however, we should understand the individual role of these three operational parameters in shaping the results of the experiments. This is the aim of the present section.

In the following sections, we will analyse the influence of each of the three parameters by isolating their effects on the simulations' results. This is done by running the simulations for different values of a given parameter, while keeping the other two parameters fixed at levels which render them neutral to the results. In our model, the three operational parameters are neutral to the results when fixed at the following values: spatial distance factor $=1$ (i.e. 
the probability of interaction between two regions is always the same, independently of the actual spatial distance between them); technological threshold $=+\infty^{15}$ (i.e. the differences in the technological capabilities of two regions, no matter how large, do not represent an obstacle for mutual learning) and the increasing returns factor $=0$ (i.e. there is no 'locally induced' growth in the technological capabilities of regions).

\subsubsection{The spatial distance factor}

The value we attach to the spatial distance (SD) factor stands for the friction imposed on interactions by space. Therefore, when the SD parameter is low, interactions are more probable between neighbouring regions, while for high values of SD the probability of interaction between two distant regions will be higher.

We infer that this parameter is crucial for defining the patterns that emerge in the map. This is illustrated in Figure 2.

Low levels of SD (when regions tend to interact only with their close neighbours) result in local polarization, intermediate levels of SD result in central polarization and high levels of SD (when spatial distance does not matter so much in defining the range of possible interactions) create a diffused pattern for the distribution of technological levels. ${ }^{16}$

Local polarization presents several clusters of yellow spots, nestling within wider clusters of red spots and blue spots, with the appearance of an unusually colourful leopard skin. ${ }^{17}$ At the periphery of these local poles, green and black regions predominate. Central polarization exhibits a single large central pole with a hierarchical pattern, similar to the one described earlier for the different regional poles. In both situations, the regions at the border of the map are relatively 'underdeveloped'. Finally, the diffused pattern approaches a random spatial distribution of technological capabilities.

The resulting patterns of spatial distribution are fairly robust. In fact, changing the other parameters, within a very broad scope, does not alter the results. The effect of raising the values of both the technological threshold and the increasing returns factor is basically one of increasing/decreasing the contrast of colours in the map (i.e. increasing/decreasing the distance between the regions' technological capabilities), with the patterns described above becoming more or less clear in the map.

In connection with the changes in those patterns, there are modifications in the convergence/divergence levels, as measured by the coefficient of variation $(\mathrm{CV})$, for different values of the SD parameter (Figure 3).

SD: 0.02

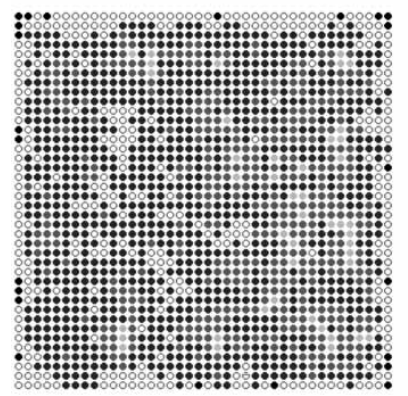

SD: 0.2

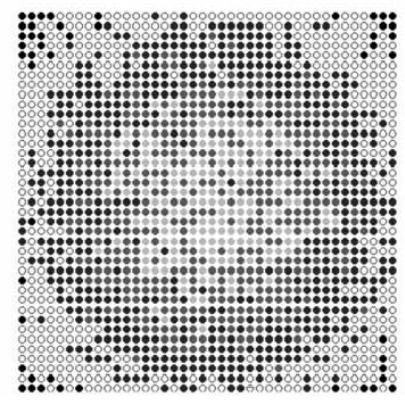

SD: 0.8

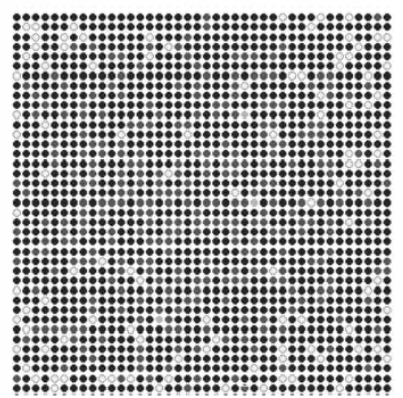

Figure 2. Distribution in space of technological capability for different values of the spatial distance (SD) parameter. 
SD: $0.02 ; \mathrm{CV}=0.287$

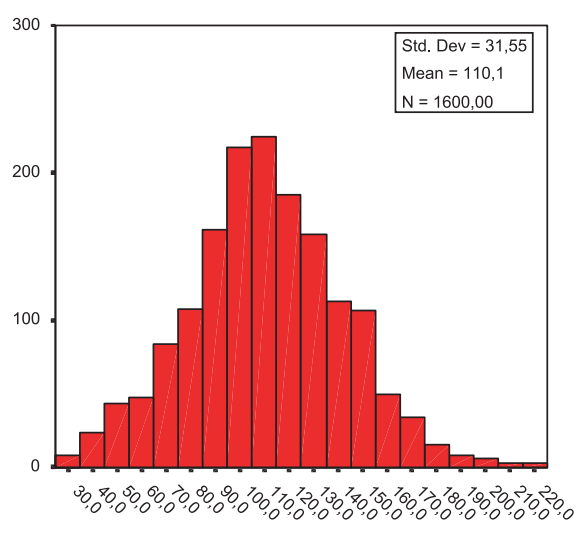

V2
SD: $0.2 ; \mathrm{CV}=0.402$

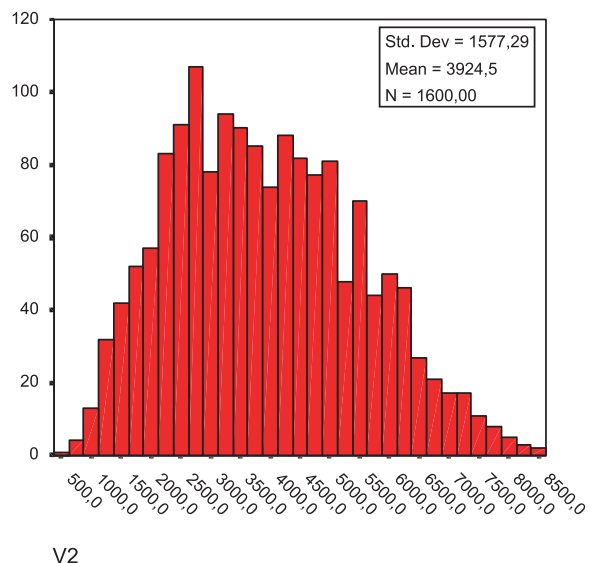

SD: $0.8 ; C V=0.179$

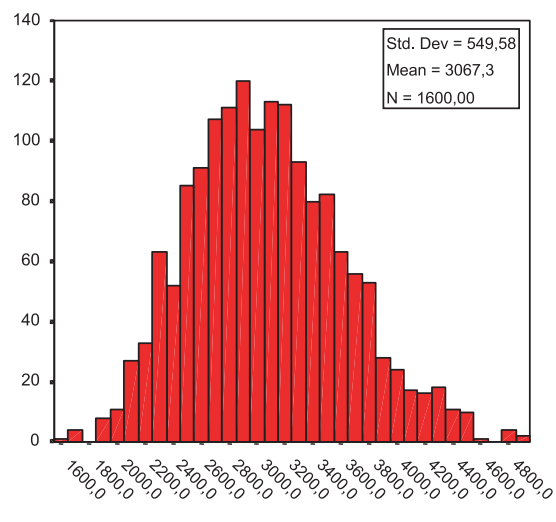

V2

Figure 3. Distribution of technological capability for different values of the spatial distance parameter.

Whatever the values of the other parameters, as long as they are kept fixed, changing the value of the SD parameter always gives rise to the following results: when we increase the value of SD from 0 up to a certain point (generally around 0.2 , where central polarization emerges), the coefficient of variation increases; after that point, divergence is reduced continuously, reaching its lowest level for $\mathrm{SD}=1$ (when there is no spatial friction being imposed on regional interactions).

This result should be kept in mind: 'reducing the friction imposed by space on interactions between regions does not necessarily result in convergence; in fact, for a certain interval, increasing the spatial scope for interactions can result in higher divergence levels (as measured by the coefficient of variation)'.

\subsubsection{The technological threshold parameter}

In the previous section, we saw that the spatial distance factor is the crucial parameter for determining the pattern of spatial distribution (local polarization, central polarization, 
diffused pattern) that one can identify in the map. Changing the values of the other parameters, within a very broad scope, does not alter those results. The effect of increasing the values for the technological threshold or for the increasing returns factor is one of increasing/decreasing the contrast of colours in the map, which corresponds to increasing/decreasing the distance between the regions' technological capabilities. Therefore, in this and the following sections, we will restrict our discussion to the analysis of both the histograms and the coefficient of variation. These are shown in Figure 4.

Increasing the value of the TT parameter means reducing the restrictions imposed on learning between regions which derive from the gap in their technological capability levels. Thus, for low levels of TT learning will only occur between regions with similar degrees of technological capabilities, while for very high levels of TT learning will occur even if two regions have very different levels of technological development.

The effect of increasing the values assigned to TT on regional convergence/divergence (as measured by the coefficient of variation) shows a similar pattern to the one identified for the SD factor. That is, 'starting from 1, the increase in the value of TT will result in growing divergence up to a certain point (usually around 1.5); after this point, the coefficient of variation will start to decrease as the value of TT grows, reaching its lowest level when technological distance no longer restricts mutual learning between interacting regions' ${ }^{18}$

From the histograms above, we can see that while it remains at fairly low levels, an increase in TT will widen the range of technological levels in the distribution, 'leaving behind' a group of regions whose capabilities are too low to learn from more advanced ones. Nevertheless, as the value of the TT parameter grows sufficiently high to allow for interaction between regions with very different levels of technological capability, the falling behind group tends to disappear.

TT: $1.25 ; \mathrm{CV}=0.810$

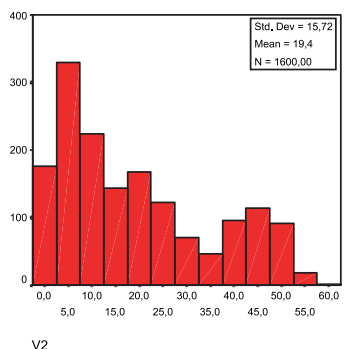

V2

TT: $4 ; \mathrm{CV}=0.349$

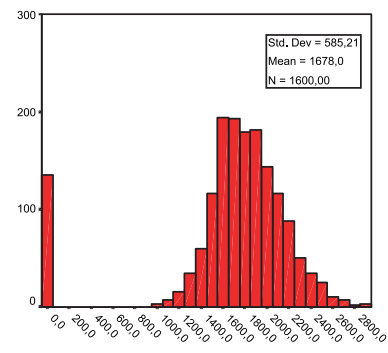

V2
TT: $1.5 ; \mathrm{CV}=0.961$

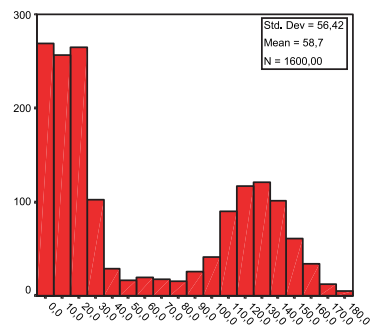

v2

TT: $6 ; \mathrm{CV}=0.177$

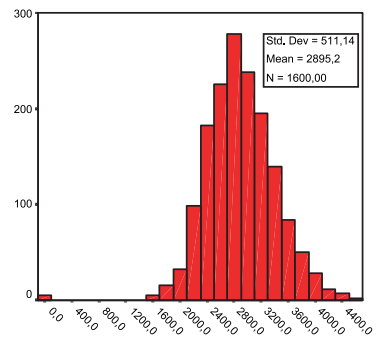

v2
TT: $3 ; \mathrm{CV}=0.445$

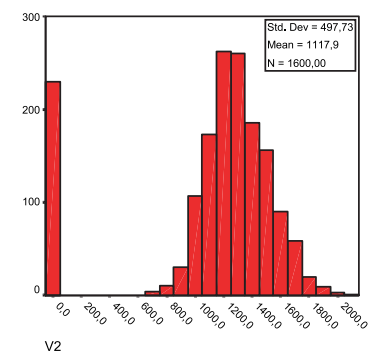

v2

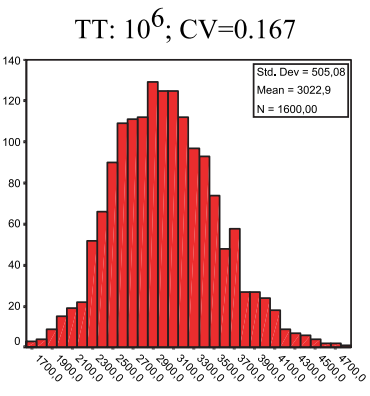

v2

Figure 4. Distribution of technological capability for different values of the technological threshold (TT) parameter. 
One should remember that in the experiments that gave rise to the outputs shown above, the other parameters (namely, the spatial distance factor and the increasing returns factor) were not seen to be exerting any influence on the results. This means that we can draw a second relevant conclusion for further discussion, which is: 'as long as there is a technological threshold restricting the possibility for mutual learning between any two regions with different levels of technological capabilities, the simulation will give rise to processes of "falling behind" for some regions'.

We will return to this conclusion later. Before this, we will analyse the effect of the third 'operational' parameter: the increasing returns factor.

\subsubsection{The increasing returns factor}

The accumulation of technological capabilities was modelled as an exponential process, in which more technologically advanced regions will ceteris paribus grow faster than the less advanced ones as a result of the increasing returns (IR) factor. Thus, the effect on convergence/divergence of increasing the values of the IR factor is straightforward: the process of convergence will become harder, giving rise to increasing values for the coefficient of variation. This is shown in Figure 5.

After looking at the effects of each of the three operational parameters (SD, TT and IR) in isolation, we will now discuss the possible results stemming from the simultaneous influence of those parameters in the simulations.

\subsection{Catching up, forging ahead and falling behind}

All the three parameters that we have been discussing tend to influence the level of convergence in technological capabilities between regions, as measured by the coefficient of variation. But they do so in different ways.

As shown above, when the value of the SD parameter increases from zero, divergence increases up to a certain point and then starts to decrease, reaching very low levels for high levels of the SD factor. The SD value for which the coefficient of variation is highest depends on the values of the other parameters. The same effect on convergence is found for increasing values of the technological threshold (TT) parameter. In contrast, increasing the values of the IR parameter always leads to higher divergence.

This allows us to infer that 'the level of divergence, as measured by the coefficient of variation, will be higher for intermediate values of the SD and the TT parameters' (i.e. $\mathrm{SD}=0.2$ and $\mathrm{TT}=1.5$, approximately) and for higher values of the IR factor. This can be seen in Figure 6a, where CV $=1.220$.

The interpretation of these results is rather intuitive. Both the SD and TT parameters fix the limits for fruitful interaction between two regions. When both parameters are at intermediate levels, regions will tend to interact only with close neighbours and will gain from those interactions only if the difference between their levels of technological capability is not too large. In this situation, more advanced regions will learn a lot with other advanced regions in their close neighbourhood, while less advanced regions will learn only with other less advanced regions nearby (and, therefore, will gain little knowledge from the interactions). The result of this process is an increase in the technological distance between those two groups of regions.

Beginning with this situation, we now bring the SD parameter up to its maximum level (i.e. we completely abolish spatial friction). This will widen the range of regions with which to interact, increasing the probability of getting in touch with some remote region with a 
IR: $0.0001 ; \mathrm{CV}=0.168$

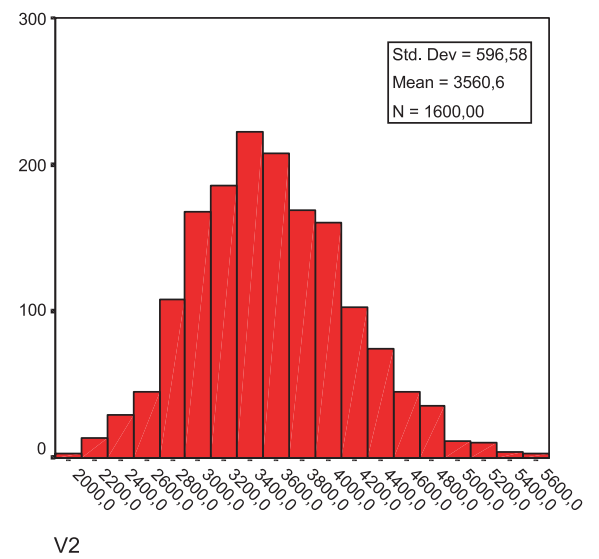

IR: $0.001 ; \mathrm{CV}=0.172$

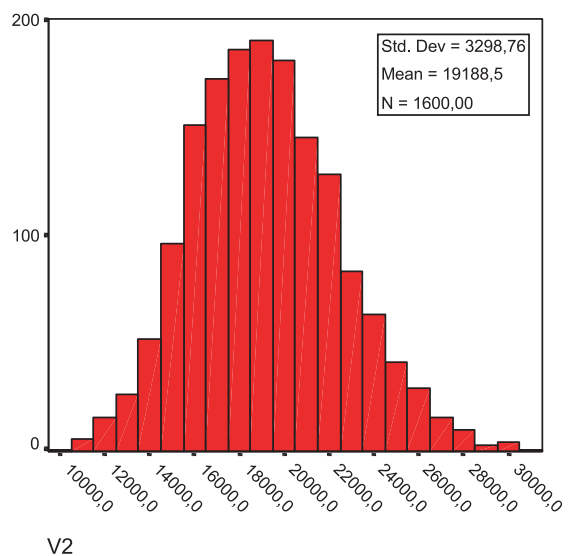

IR: $0.003 ; C V=0.183$

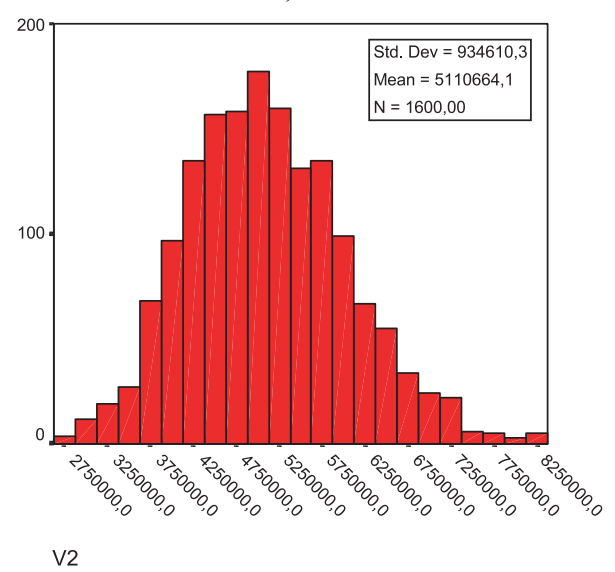

Figure 5. Distribution of technological capability for different values of the increasing returns (IR) factor.

higher level of technological development (allowing for fruitful learning) but not much higher (i.e. the learning process is still conditioned by the technological distance threshold between the two regions). Widening the spatial opportunities for learning leads to a decrease in the dispersion of technological levels among regions.

Nevertheless, a reduction in spatial friction, no matter how great, is not sufficient per $s e$ to eliminate all the sources of divergence. Insofar as there is a strong threshold condition defining the differences in technological capabilities above which two regions are not able to learn from each other, convergence between regions will be modest. Furthermore, when the technological threshold is at intermediate levels, even with no spatial friction blocking interactions (nor, for that matter, 'locally induced' growth), the development process will lead to the formation of a falling behind group of regions. These are economies which are too backward in technological capabilities to learn form the more advanced group. Such a fact is shown in the histogram in Figure 6b.

Now, we will observe what happens if we fix the SD factor at intermediate levels and, at the same time, increase the value of the technological threshold (histogram in Figure 6c 
(a) SD:0.2; TT:1.5; CV:1.220

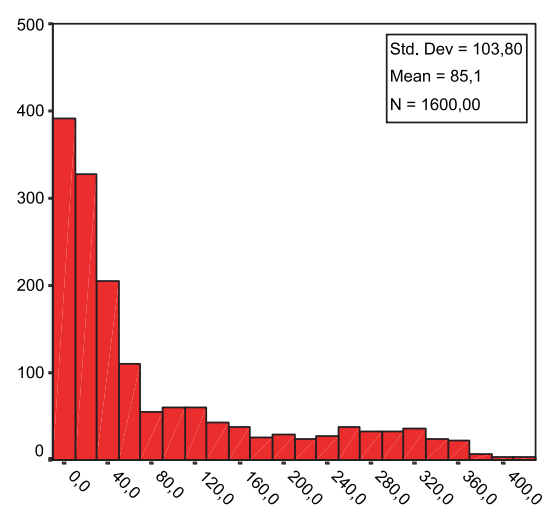

V2

(c) SD:0.2; TT:10 $0^{6}$ CV:0.415

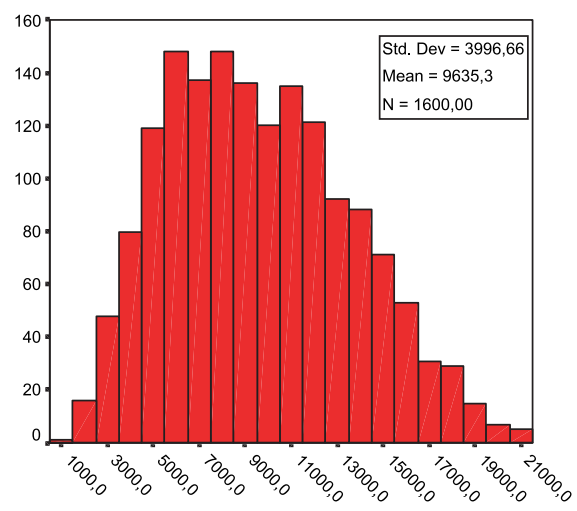

$\mathrm{V} 2$ (b) SD:1; TT:1.5; CV:1.020

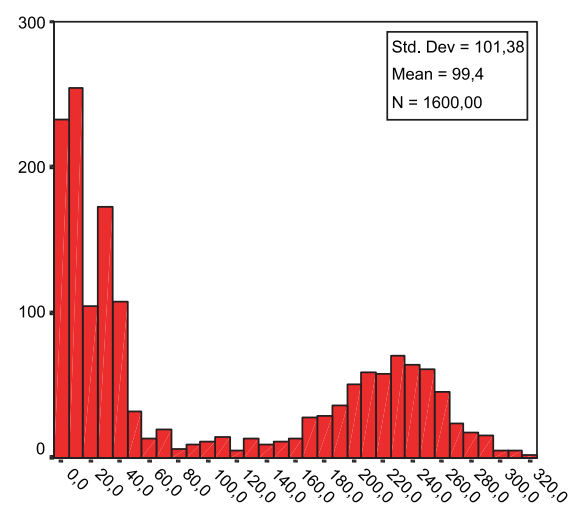

V2

(d) SD:0.8; TT:10; CV:0.169

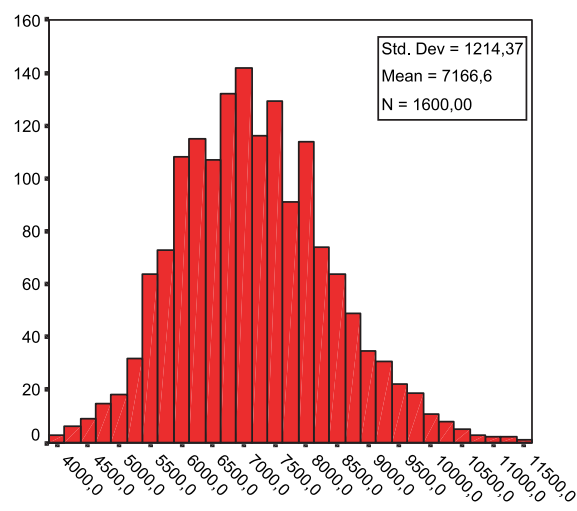

V2

Figure 6. Distribution of technological capability for different values of the spatial distance and technological threshold parameters. ${ }^{22}$

above). As we saw before, while remaining at intermediate levels, an increase in TT will widen the range of technological levels in the distribution, 'leaving behind' a group of regions whose capabilities are too narrow for them to learn from more advanced regions. Nevertheless, as the value of the TT parameter grows sufficiently high to allow for interaction between regions with any level of technological capability, the falling behind group tends to disappear, even though the coefficient of variation remains at moderate levels $(\mathrm{CV}=0.415$, in this example).

The last case pictured above (histogram in Figure 6d) is one where neither spatial nor technological distance imposes any restrictions on the learning processes between regions. This is the case in which the level of convergence is higher. As could be expected from the discussion above, situations that are close to total convergence only occur for high levels of both the SD and TT parameters.

The low levels of divergence that remain $(\mathrm{CV}=0.169)$ are due to the influence of the other parameters. First of all, in the experiments above, the increasing returns factor was fixed at 0.0005 . Lower levels of the IR factor would decrease the value of the 
coefficient of variation. But, even with IR $=0$, total convergence may not occur. This is so because interactions are modelled here as random events. Relative growth is therefore also a matter of chance. ${ }^{19}$ This brings us to the question of the relevance of history in convergence/divergence processes, to which we turn next.

\subsection{The relevance of history}

In the preceding sections, we analysed the forces behind convergence/divergence trends, starting from a specific initial distribution of technological capabilities among regions. This distribution is achieved by attaching a value to a random generator seed (see variable $r$ in Section 3). The value we choose for this seed will also affect the process of selecting the pair of regions that will interact at each time period. This poses the question of whether the results discussed in the preceding sections are sensitive to both the initial distribution of technological capabilities among regions and the ensuing selection process for interaction at each time period. In other words, we should investigate to what extent history matters for the results we obtain from our experiments.

To analyse the relevance of the initial distribution of capabilities among regions and the selection mechanism in the process of convergence/divergence, we ran the same experiments, but in this case starting from a homogeneous distribution of technological capabilities, where every region has an initial technological level of 5 (colour blue). ${ }^{20}$ The resulting maps are shown in Figure 7.

The patterns of change in the level of convergence (measured by the coefficient of variation; not shown) that are caused by increasing values of the three parameters analysed earlier are not sensitive to changes in the initial conditions: CV increases for low to intermediate levels of the spatial distance factor (SD) and then decreases as the values of this factor get higher; the same happens for the technological threshold factor; finally, raising the values of the increasing returns (IR) factor always leads to an increase in the coefficient of variation.

In addition to this, the patterns of spatial distribution seem to be fairly robust in response to changes in the initial distribution of technological capabilities. As Figure 7 clearly shows, even when all regions start with a common technological level, low levels of SD result in local polarization, intermediate levels of SD result in central polarization and high levels of SD create a diffused pattern for the distribution of technological levels.

This is not to say that the overall convergence/divergence trends are completely neutral in relation to historical conditions (as defined in the introduction to this section). This is

SD: 0.02

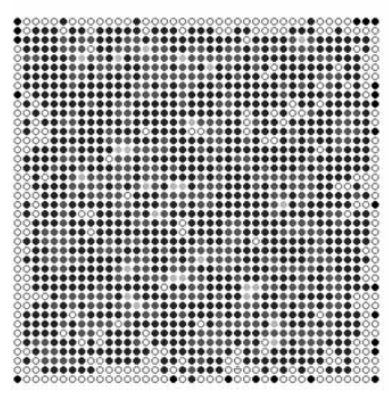

SD: 0.2

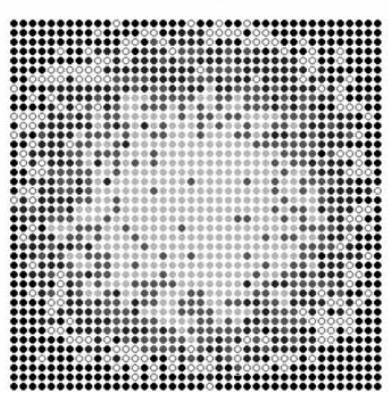

SD: 0.8

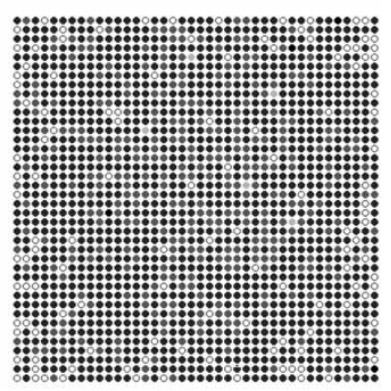

Figure 7. Results of experiments starting from a homogeneous distribution of technological capabilities. 
true at the aggregate level, but not necessarily in relation to the specific regions that are 'catching up', 'forging ahead' or 'falling behind' in each case. For both low and high values of the SD parameter (say, between 0 and 0.2 , and between 0.7 and 1, respectively), changes in the initial distributions and/or different random generator seeds can mean that regions that were 'losers' in one case become 'winners' in another (and vice versa). On the other hand, for intermediate levels of the SD factor, the central regions will always tend to win, no matter what may be the initial distribution or the random generator seed.

These results can be understood in the light of the following reasoning. When interactions between regions are spatially concentrated in several poles, the specific initial location of regions with both high and low levels of technological development will affect the shaping of the final distribution of technological capabilities in the map. Contrarily, if the central polarization forces are very strong, then the initial position of each region in terms of technological developments does not really matter. In that case, growth potential will always end up being stronger in the centre.

\subsection{The relevance of space configuration}

Until now, we have been working with a given space configuration, corresponding to a $40 \times$ 40 matrix of regions. In reality, whatever sort of space is being considered (geographical, cultural, economic, etc.), it is difficult to find a situation where regions are organized in such fashion. We therefore tried to investigate the consequences of running the simulations with different spatial configurations. This was done by attaching the value 0 to some of the cells in the map, thereby considering these as representing 'non-regions' or 'uninhabited' regions.

Let us now look at the effects of changing the spatial configuration of the map (Figure 8).

Starting from the figures on the right side, we can conclude that for high values of the spatial parameter, different spatial configurations do not seem to give rise to markedly distinct

SD: 0.02
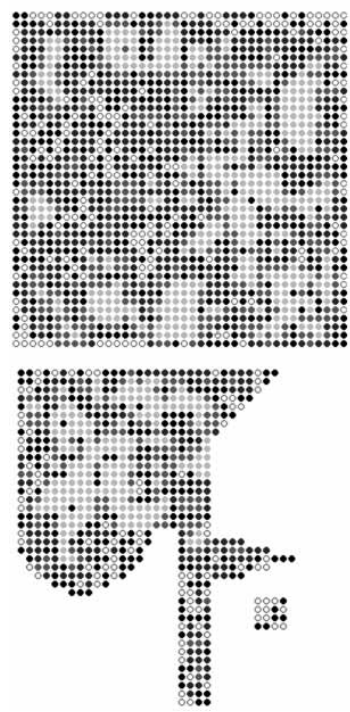

SD: 0.2
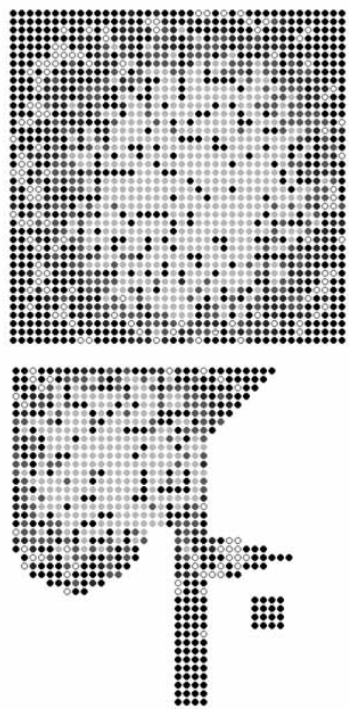

SD: 0.8
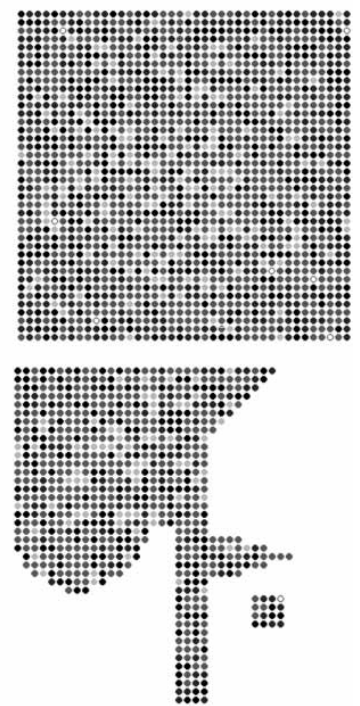

Figure 8. Distribution of technological capability for different spatial configurations. ${ }^{23}$ 
spatial distribution patterns for technological capabilities. ${ }^{21}$ The same is not, however, true for the other situations pictured above.

In the intermediate case, when regions tend to interact with more distant ones, but when space still causes high friction in relation to the probable length of such contacts, we can identify the already noticed tendency towards central polarization. But in the configuration at the bottom, the more developed dominant pole is clearly biased towards the 'northwest' (where we can find a large mass of neighbouring regions), and it does not reach regions that are part of the central pole in the map at the top. The enlargement of the developed centre is slowed down, or even blocked, whenever the connections with some regions are reduced or absent and the mass of regions in the respective neighbourhood is low (as in the cases of the 'peninsula', the 'island' and the tip of the 'beak' represented in the bottom picture).

The effect of specific spatial configurations on the distribution of technological capabilities in the map is also very pronounced for low values of the SD parameter. It is possible (see the bottom left map in Figure 8) to identify a clear tendency for local polarization effects, which happen when 'critical masses' of regions exist next to each other (e.g. the 'northwest' concentration, and the base of the 'beak' at the centre). These results would be even more noticeable for higher values of the technological threshold parameter - in such cases we would hardly find any advanced regions (i.e. clearer spots in the map) within the 'peninsula' (or the 'island').

These results are explained by the fact that regions grow faster when they are able to interact with other regions, especially if the latter are more developed. If we fix the SD factor at low levels, this means that regions are only able to learn from their close neighbours. Therefore, the technological capabilities of each region will grow faster when (i) there are many regions in the surrounding area (increasing the possibility of finding another one to interact with), (ii) these surrounding regions have relevant capabilities to exchange (increasing the growth associated with diffusion effects) and (iii) there are no technological barriers to learning from other regions (increasing once again the number of regions with which useful interactions can be established). For low levels of the SD factor and high levels of the TT parameter, regions located in the core of 'continental masses' will therefore benefit in terms of increased technological capabilities.

\section{Conclusion}

In Section 2 of the paper, we contrasted the main theoretical approaches to economic convergence analysis and briefly referred to the major findings of some recent empirical literature on this issue. From this discussion, we were able to draw three main indications. The first one is that convergence is normally associated with a need on the part of catchingup economies to meet several prerequisites, not only in terms of the accumulation of factors, but also in structural and institutional terms. Secondly, it is also clear that the catching-up economies can derive advantages from their external contacts. But, in order to maximize the benefits from interactions, they need both to have a minimal level of technological capability (i.e. the gap should not be too large for communication to occur) and to be relatively 'close' to the leading economies (with 'closeness' here referring not only to geographical distance, but also to economic, institutional or cultural distances). Thirdly, economic convergence is not a necessary outcome of interactions or integration between economies. On the contrary, there are good reasons to believe that for certain historical and technological conditions the leading economies may increase their lead as a result of local increasing returns and 'cumulative causation' processes. 
The simulation model which we put forward in Section 3 of the paper reflects these basic indications from the theoretical discussion. Namely we assume that the laggard economies do not benefit automatically from the knowledge produced by the leading economies, as if it were a kind of manna from heaven. In contrast to conventional neo-classical approaches, we assume that the catching-up economies need to develop local efforts and build up minimal levels of capability in order to absorb the knowledge spilling over from the more advanced economies.

The simulation exercise carried out aimed at understanding the factors underlying the dynamics of regional technological capability accumulation and the overall trends in terms of convergence/divergence. Two competing processes are involved in understanding the evolution of technological capability (TC), the dependent variable of the model. Process (a) leads to a divergent trend in terms of TC variation, whereas process (b) introduces compensating factors, associated with knowledge diffusion, that contribute to convergence. Process (a) refers to the domestic creation of TC through local accumulation efforts. Synergies arise in this accumulation of tangible and intangible factors, giving rise to increasing returns in the evolution of TC. In process (b), TC depends on the diffusion of knowledge which happens whenever any two regions within a given technological distance interact. The amount of knowledge that spills over in that interaction is related to the technological level of the interacting partners.

The implementation of the simulation, over a given period of time and with varying values for the critical parameters, produced graphical (in the form of a map with coloured dots) and statistical (histogram, coefficient of variation of TC) outputs, which were thoroughly examined in Section 4 of the paper. It was possible for several conclusions to be drawn from this exercise.

First, it became clear that situations of near total convergence only occur when: (i) mutual learning is not restricted by regional differences in the levels of TC; (ii) spatial distance does not impose any friction on interactions, thus making it as probable for two distant regions to interact as for two close ones; (iii) there are no increasing returns in the process of technological development. If these three conditions are not simultaneously met, total convergence will not be achieved.

Secondly, reducing the friction imposed by space on interactions between regions does not necessarily result in convergence. In fact, for a certain interval, increasing the spatial scope for interactions can result in higher divergence levels (as measured by the coefficient of variation). By the same token, reducing the technological threshold, which defines the gap of technological levels above which no mutual learning is possible, does not necessarily result in convergence. Within a certain interval, making it easier for two regions with different levels of technological development to learn from each other can result in higher divergence levels (as measured by the coefficient of variation). The level of divergence, as measured by the coefficient of variation, is therefore highest when regions interact, but the distance restriction does not allow relationships to be developed with too distant neighbours, while the technological threshold dictates that they have relatively similar technological levels. Furthermore, when the technological threshold is at intermediate levels, even with no geographical friction blocking interactions, the growth process will lead to the formation of two separate groups of regions: one 'forging ahead' group and one 'falling behind' group.

Thirdly, it is clear that regardless of the initial distribution of technological capabilities or the way in which the random process of interactions among regions proceeds, when spatial friction is at intermediate levels (i.e. interactions are not restricted to the closest neighbours, but still the probability of one region interacting with a very distant one is not 
the same as the probability of its interacting with a less distant one), the technologically advanced regions will always tend to be located in the centre (or within agglomerations of regions). However it is also clear that under certain conditions the initial distribution and the ensuing pattern of interactions are relevant ('history matters'). This is the case when the spatial friction is either relatively low or high. In these circumstances, regions that could be 'losers' for a given initial distribution can become 'winners' for another one.

Finally, when spatial distance imposes high friction on interactions between regions and when regions do not have to be very similar in their levels of technological capabilities to learn from each other, regions at the core of 'continental masses' benefit in terms of increased technological capability.

To finalize, we should like to point out some avenues of work which might be derived from both the testing of this model and the analysis of the outputs that were carried out in this paper. In the near future, the exploration of the results is due to be completed, namely through the parametrization of the distance factor. The model can still be developed by trying to overcome the following already diagnosed shortcomings:

- the idea of a constant and single increasing returns factor seems rather unrealistic;

- the implementation of diffusion is too simplistic - the rate of diffusion should be related to the technological distance between regions (and the diffusion parameter should not be a constant);

- real space is not unidimensional, but multi-dimensional spaces probably cannot be aggregated;

- time does not have a clear interpretation in the model (the different time scales are arbitrary);

- the analysis of the average values of the dependent variable of the model (TC) will also be given attention in our future work.

In addition to these shortcomings, there is a yet more fundamental problem stemming from the fact that history is also a consequence of deliberate action and power relations (i.e. it is not only based on postulated initial conditions and random processes of interaction). The production and use of knowledge depends on institutions that may either facilitate or hinder the underlying processes. When perceived as hindrances, such institutions are amenable to change and the players do in fact act to transform them.

As put by North, institutions (and institutional details) are crucial to understand processes of economic change and so is 'the intentionally of the players enacting institutional change' $(2005,3)$. We are still a long way from being able to incorporate those elements in any formal model. Notwithstanding, modelling exercises may still be useful as heuristic aids to theory development, especially when they are thought provoking and controversial - as we believe is the case with the present one.

\section{Acknowledgement}

The support from the EU TSER programme (contract SO E2-CT98-2047) is acknowledged.

\section{Notes}

1. Even in the absence of factor mobility, neo-classical trade theory predicts that free trade will ensure the most efficient specialization in production (in accordance with factor endowments), thus leading to convergence.

2. These arguments are in line with Alfred Marshall's theory of agglomeration economies and have been re-interpreted and extended by modern economic geography, among other disciplines. 
3. The important role of various pre-requisites had already been noted in the Economic Development literature of the 1950s and 1960s, which emphasized the need for a structural adjustment of the economy towards newer, more productive, sectors.

4. Most importantly, for our concerns in this paper, attempts have been made to cross these developments with the fertile field of economic geography and its emphasis on the role of agglomeration economies. Such economies influence the location decisions of individual firms in relation to specific industrial centres, therefore adding elements of cumulative causation to the trajectories of growth and convergence among economies (Caniëls and Verspagen 1999).

5. The econometric analysis of convergence trends in terms of GDP per capita in Europe, presented in Cappelen, Fagerberg and Verspagen (1999), shows that R\&D expenditures are not positively correlated with a faster catching up of the less-favoured European regions. This same analysis shows that such expenditures are only positively correlated with the growth of GDP per capita for a smaller 'club' of 29 more advanced European regions, while they are negatively correlated for the remaining 76 regions. As this same paper puts it, 'innovative efforts in the form of investments in R\&D appear to be most efficient in advanced regions'.

6. This observation is in keeping with traditional diffusion theory. According to this, one of the critical conditions for adoption is the size of the adopting agent, which for a given characteristic needs to be above a certain threshold level (David 1969, Davies 1979).

7. Based on the analysis of patent citations, these authors show that, for the USA, citations are more likely to come from the same state as the cited patents when compared with a 'control frequency'.

8. Using European Patent Office data, these authors conclude that the results of research carried out locally, as can be seen in the patterns of patents' citations, tend to be used more frequently by local firms than by firms located in other regions. Moreover, a similar pattern for citations is to be found between regions of the same country.

9. In the simulations for which the results are to be presented dim was set at 40 .

10. If region $k l$ is an empty spot, no interaction occurs in the time step. If non-admissible values of $k$ and $l$ are obtained, i.e. if $k \notin[1, \operatorname{dim}]$ or $l \notin[1, \operatorname{dim}]$, or $k=i$ and $l=j$, a new region $i j$ is chosen and the process of finding its interacting partner is repeated.

11. The required prerequisite that the technological gap should not be above a given technological threshold (TT) is in line with the idea of 'technological congruence' discussed in Section 2.

12. Note that this number of time steps might also be parametrized.

13. One should note, nevertheless, that the model includes other implicit parameters that are taken as fixed but which can be changed, as was suggested previously.

14. The option for the latter value is equivalent to eliminating the restrictions in terms of differences in technological capabilities that are imposed on learning between any two regions.

15. As mentioned previously, the highest value we actually attached to the TT parameter was 10 million, since this was enough for the stated purposes in the simulations we ran.

16. The precise meaning of low, intermediate and high levels of the SD parameter depends on the values attached to the other parameters, and it is always difficult to define this meaning, since there is a continuum between the shapes in Figure 3. Roughly speaking, SD values between 0 and 0.05 result in local polarization, between 0.05 and 0.7 in central polarization and between 0.7 and 1 in a diffused pattern.

17. When the maps are printed in black and white, part of the information is lost. When the figures are printed in grey scale, as a general rule, clearer spots correspond to higher comparative levels of TC and darker spots to lower levels of TC; the exception is the green spots (representing the next to last comparative level of TC), which is represented as a white spot encircled by a dark line, in order to obtain the necessary contrast with the darkest spots.

18. In the case pictured above, a value of 10 million for TT is sufficient to ensure that this condition is met.

19. For these parameter values, total convergence is an asymptotic property of the model, but it will not occur within a given time period.

20. We also ran experiments using other initial distributions and different random generator seeds. All of the following results hold true.

21. Note that, since, even in this situation, we did not completely abolish the influence of spatial distance; the regions within the 'island' that are facing the 'continent' are more developed than the others.

22. In the experiments, the results of which are shown, we attached the value 0.0005 to the increasing returns factor. 
23. In the experiment whose results are shown in this figure, we attached the following values to the 'operational' parameters: SD: 0.02, 0.2 and 0.8; TT: 2.5; IR: 0.0005.

\section{References}

Abraham, F., and P. van Rompuy. 1995. Regional convergence in the European Monetary Union. Papers in Regional Science 74, no. 2: 125-42.

Abramovitz, M. 1986. Catching up, forging ahead, and falling behind. Journal of Economic History XLVI, no. 2: 385-406.

Armstrong, H. 1995. Convergence among regions of the European Union, 1950-1990. Papers in Regional Science 74, no. 2: 143-52.

Arrow K.J. 1962. Economic welfare and the allocation of economic resources for invention. In Collected papers. 1984 ed. Vol. 4, 104-19. Oxford: Basil Blackwell.

Barro, R., and X. Sala-i-Martin. 1991. Convergence across states and regions. Brooking Papers on Economic Activity 1: 107-82.

Caniëls, M., and B. Verspagen. 1999. Spatial distance in a technological gap model. ECIS Working Paper 99.10, Eindhoven Centre for Innovation Studies.

Cappelen A., J. Fagerberg, and B. Verspagen. 1999. Lack of regional convergence. Paper presented at the EAEPE 1999 conference, 4-7 November in Prague.

David, P. 1969. A contribution to the theory of diffusion. Memorandum No. 71, Research Centre in Economic Growth, Stanford University.

Davies, S. 1979. The diffusion of process innovations. Cambridge: Cambridge University Press.

Fagerberg, J. 1987. A technology gap approach to why growth rates differ. Research Policy 16, no. 2: 87-99.

Fagerberg, J. 1994. Technology and international differences in growth rates. Journal of Economic Literature 32: 1147-75.

Fagerberg, J., B. Verspagen, and M. Caniëls. 1997. Technology gaps, growth and unemployment across European regions. MERIT Working Paper, Maastricht.

Gerschenkron, A. 1962. Economic backwardness in historical perspective. Cambridge, MA: Harvard University Press.

Griliches, Z. 1992. The search for R\&D spillovers. Scandinavian Journal of Economics 94 (Suppl.): 2-47

Jaffe, A. 1996. Economic analysis of research spillovers: Implications for the advanced technology program. Gaithersburg, MD: NIST Grant/Contract Report 97-708, US Department of Commerce, Technology Administration, National Institute of Standards and Technology, December 1996.

Jaffe, A., M. Trajtenberg, and R. Henderson. 1993. Geographic localisation of knowledge spillovers as evidenced by patent citations. Quarterly Journal of Economics 108, no. 3: 577-594.

Kaldor, N. 1957. A model of economic growth. Economic Journal 67, no. 268: 591-624.

Klenow, P., and A. Rodríguez-Clare. 2005. Externalities and growth. In Handbook of economic growth, ed. P. Aghion and S. Durlauf, 817-61. Amsterdam: North Holland Press.

Maurseth, P., and B. Verspagen. 1998. Knowledge spillovers in Europe and its consequences for systems of innovation. MERIT Working Paper 2/98-024, Maastricht.

Nelson, R.R. 1959. The simple economics of basic scientific research. Journal of Political Economy LXVII: 297-306.

Neven, D., and C. Gouyette. 1995. Regional convergence in the European community. Journal of Common Market Studies 33, no. 1: 47-65.

North, D. 2005. Understanding the process of economic change. Princeton: Princeton University Press.

Pavitt, K. 1985. Technology transfer among the industrially advanced countries: An overview. In International technology transfer: Concepts, measures and comparisons, ed. N. Rosenberg and C. Fristack, 3-23. New York: Praeger.

Posner, M.V. 1961. International trade and technical change. Oxford Economic Papers 13: 323-41.

Romer, P. 1986. Increasing returns and long-run growth. Journal of Political Economy 94: 1002-37.

Verspagen, B. 1991. A new empirical approach to catching up or falling behind. Structural Change and Economic Dynamics 2, no. 2: 359-80. 